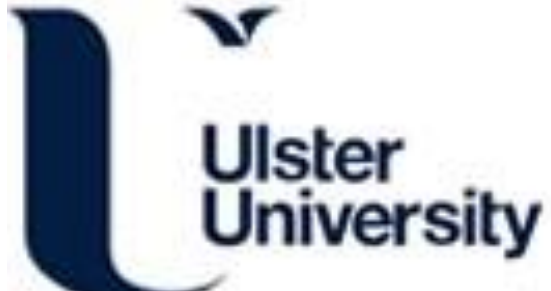

\section{Ontology-based Activity Recognition Framework and Services}

Chen, L., Nugent, CD., \& Rafferty, J. (2013). Ontology-based Activity Recognition Framework and Services. In IIWAS '13 Proceedings of International Conference on Information Integration and Web-based Applications \& Services Association for Computing Machinery. https://doi.org/10.1145/2539150.2539187

Link to publication record in Ulster University Research Portal

\section{Published in:}

IIWAS '13 Proceedings of International Conference on Information Integration and Web-based Applications \& Services

\section{Publication Status:}

Published (in print/issue): 01/12/2013

DOI:

$10.1145 / 2539150.2539187$

\section{Document Version}

Publisher's PDF, also known as Version of record

\section{General rights}

Copyright for the publications made accessible via Ulster University's Research Portal is retained by the author(s) and / or other copyright owners and it is a condition of accessing these publications that users recognise and abide by the legal requirements associated with these rights.

\section{Take down policy}

The Research Portal is Ulster University's institutional repository that provides access to Ulster's research outputs. Every effort has been made to ensure that content in the Research Portal does not infringe any person's rights, or applicable UK laws. If you discover content in the Research Portal that you believe breaches copyright or violates any law, please contact pure-support@ulster.ac.uk. 


\section{Ontology-based Activity Recognition Framework and Services}

\author{
Liming Chen \\ University of Ulster \\ Shore Road, Newtownabbey \\ Northern Ireland. BT37 0QB \\ +442890368837 \\ I.chen@ulster.ac.uk
}

\author{
Chris Nugent \\ University of Ulster \\ Shore Road, Newtownabbey \\ Northern Ireland. BT37 OQB \\ +442890368330 \\ cd.nugent@ulster.ac.uk
}

\author{
Joseph Rafferty \\ University of Ulster \\ Shore Road, Newtownabbey \\ Northern Ireland. BT37 OQB \\ +442890368840 \\ rafferty-j@email.ulster.ac.uk
}

\begin{abstract}
This paper introduces an ontology-based integrated framework for activity modeling, activity recognition and activity model evolution. Central to the framework is ontological activity modeling and semantic-based activity recognition, which is supported by an iterative process that incrementally improves the completeness and accuracy of activity models. In addition, the paper presents a service-oriented architecture for the realization of the proposed framework which can provide activity context-aware services in a scalable distributed manner. The paper further describes and discusses the implementation and testing experience of the framework and services in the context of smart home based assistive living.
\end{abstract}

\section{Categories and Subject Descriptors}

I.2.1 [Artificial Intelligence]: Applications and Expert Systems industrial automation, decision support, health.

\section{General Terms}

Algorithms, Management, Measurement, Performance, Design,

\section{Keywords}

Activity recognition, service based computing, ontology, smart home, ambient assisted living.

\section{INTRODUCTION}

With the rapid development of low-power low-cost miniaturized sensing technologies, and wired and wireless communication techniques ubiquitous / pervasive computing [1] has evolved from a vision towards a reality which enables a new paradigm of human-centric computer interaction in an environment to allow thorough integration of everyday objects and activities with information processing. This has led to a new breed of applications based on the conception of smart environment where miniaturized computing devices work in concert to support people

Permission to make digital or hard copies of all or part of this work for personal or classroom use is granted without fee provided that copies are not made or distributed for profit or commercial advantage and that copies bear this notice and the full citation on the first page. To copy otherwise, or republish, to post on servers or to redistribute to lists, requires prior specific permission and/or a fee.

iiWAS2013, 2-4 December, 2013, Vienna, Austria

Copyright 2013 ACM 978-1-4503-2113-6/13/12 ...\$15.00 in carrying out their daily working and living activities in an easy, natural and personalized way. A typical real-world example of such an environment is a "Smart Home" within which the daily activities of its inhabitants, usually the elderly or disabled, are monitored and analyzed so that personalized context-aware assistance can be provided. Others examples include intelligent meeting rooms, conference centers, hospitals and shopping malls, intelligent cars, to name but a few.

Context awareness has been the core of ubiquitous computing and a center of research in ubiquitous computing paradigm and applications. In order to make a system context-aware, it is necessary to 1) acquire contextual information usually using sensing technologies, 2) model and represent contextual information, 3) store and manage contextual information, and 4) retrieve and interpret the context. These requirements have invoked considerable research on context modeling, representation and reasoning as well as technological frameworks and middleware for context management [2].

Context has been studied in terms of the level of abstraction, namely low-level context derived directly from individual sensor readings and high-level context built upon aggregation and fusion of multi-modal sensor data from multiple sources. Activity is a type of high-level context which play a significant role in a number of application domains, e.g. surveillance-based security, context-aware computing in pervasive and mobile environments and ambient assisted living in smart homes. As a result, activity modelling and recognition has been under vigorous investigation and activity based computing has received growing attention. Nevertheless, current research has mainly focused on componentlevel methods, techniques and technologies, e.g. sensor data segmentation, activity modelling and activity recognition. Little work has been undertaken towards the provision of system-level solutions, e.g. the integrated framework and technology infrastructure that are reusable, applicable and scalable to real world problem solving.

This paper introduces an ontology-based integrated framework for activity context modeling, recognition and activity model evolution. Central to the framework is ontological activity modeling and semantic-based activity recognition, which is supported by an iterative process that incrementally improves the completeness and accuracy of activity models. In addition, the paper presents a service-oriented architecture for the realization of the proposed framework which can provide activity context-aware services in a scalable distributed manner. The paper further describes and discusses the implementation and experience of the 
framework and services in the context of smart home based assistive living. It is worth noting that the focus of the paper is not on the enabling models, algorithms and methods of the underlying component but the overall framework, interplay and iterative process of constituent components as well as implementation and deployment architecture.

The remainder of the paper is organized as follows. Section 2 reviews related work. Section 3 describes the ontology-based integrated framework for activity context aware system. Section 4 presents a service oriented architecture for the proposed framework. Section 5 outlines the implementation details of both a standalone and a SOA based system. Section 6 presented testing results of the framework and services in the context of smart home based assistive living. The paper is concluded in section 7 .

\section{RELATED WORK}

Activity context modelling and recognition can be generally classified into two categories of approaches based on the way activities are monitored. The first is based on the use of visual sensing facilities, e.g., camera-based surveillance systems, to monitor an individual's behavior and environmental changes [3] [4]. The approaches in this category exploit computer vision and image analysis techniques to infer visual observations for pattern recognition [5] [6]. The second category is based on the use of emerging sensor network technologies for activity monitoring. In these approaches, sensors can be attached to an user under observation - namely wearable sensors, or objects that constitute the activity environment - namely dense sensing. Wearable sensors often use inertial measurement units and RFID tags to gather an user's behavioral information [7]. This approach is effective for recognizing physical movements such as physical exercises [8]. In contrast, dense sensing infers activities by monitoring human-object interactions [9] through the usage of low-power and low-cost sensors.

With the dense sensing paradigm, activity context modelling and recognition can be performed in several strands with the fundamental differences relating to the manner in which activities are modeled and represented. One strand is referred to as the generative approach, which attempts to build a complete description of the input or data space, usually with probabilistic analysis methods such as Markov models [26] and Bayesian networks [11] for activity modelling. These methods incorporate an inhabitant's preferences by tuning the initial values of the parameters of the probabilistic models. An alternative approach is referred to as the discriminative approach, which only models the mapping from inputs (data) to outputs (activity labels). Discriminative approaches include many heuristic (e.g. rulebased) approaches, for example, neural networks, linear or nonlinear discriminant learning. They use machine learning techniques to extract Activities of Daily Living (ADL) patterns from observed daily activities, and later use the patterns as predictive models [13] [21].

The third strand, i.e., the knowledge-driven approach, uses logical formalisms, for example the event calculus [14], lattice theory [12], description logics [27] [16] for representing ADL models and conducts activity explanation and prediction through deduction or abduction reasoning. Comparing to the above two data-centric approaches, Knowledge-driven approaches solves the cold-start problem, i.e. they do not require pre-existing large scale dataset for model learning. It is semantically clear and elegant in reasoning as the approach is based on the solid foundation of formal knowledge representation theories. The major weaknesses are that the pre-defined models are static and incomplete due to the limited knowledge of individual experts.

Significant progress has so far been made in activity monitoring, modelling and recognition pushed by the prevalence and advance of the latest sensing and wireless communication technologies, and pulled by the emerging wave of context-aware applications within pervasive environments, such as ambient assisted living. Nevertheless, these advances are mainly at component level focusing on sensing devices, modelling, data processing algorithms and reasoning mechanisms. Little work has been undertaken towards generic frameworks and systematic technology infrastructure [20] that can support activity context aware systems in terms of scalability, reusability and applicability.

The work presented in this paper follows the knowledge approach which uses ontological modelling and reasoning for activity context modelling and recognition. Nevertheless, it goes beyond the state of the art of ontology-based activity modelling and recognition by providing an integrated systematic approach to address the drawbacks of model incompleteness and rigidity, thus making the approach realistically feasible, applicable and reusable. Furthermore, this paper introduces a service-oriented framework for the realization of the proposed systematic approach.

\section{A GENERIC ACTIVITY RECOGNITION FRAMEWORK}

We have developed an ontology-based integrated framework for activity modelling, evolution and recognition as depicted in Figure 1. The framework aims to co-ordinate and streamline the core activities relating to activity recognition within AAL towards a systematic solution that is scalable and applicable to real world use cases such as smart homes. The underlying idea to the framework is that extensive a priori and domain knowledge are available in AAL, and formal knowledge modelling, representation and reasoning techniques can be used to develop a top-down approach to activity modelling and recognition. Ontologies have been adopted as the unified conceptual backbone for modelling, representing and inferring activity context, user activity profiles and contexts. Ontology engineering along with the latest semantic technologies, tools and APIs such as ontology editors, languages, reasoners and semantic repositories, provide the enabling technologies for the framework.

Central to the framework is an iterative process of activity modelling, activity recognition and model learning that incrementally improves the completeness and accuracy of activity models, thus leading to increasing performance of activity recognition. As can be seen in Figure 1, the process consists of four tasks, namely Ontological Activity Modelling, Semanticbased Activity Recognition, Activity Learning and Activity Model Evolution. Each task plays a distinguishable role in the process, and is supported by a number of supportive components. The goal of the Ontological Activity Modelling task is to create a number of formal knowledge models for the specific application scenarios within smart environments through the Ontology Engineering component. These models include the initial activity ontologies, user activity profiles, context ontologies, and learning and evolution rules. The initial activity models are stored in the "Seed" Activity Ontologies component and used as the starting active activity models for activity recognition. The user activity 
profile refers to the specific way a user performs activities which is used to provide personalized assistance.

The Semantic-based Activity Recognition task performs ontological classification using semantic subsumption reasoning to realize continuous progressive activity recognition in real time. It takes as inputs the active activity models and a context generated by the Context Generator and Context Ontologies components. The recognition task generates a sequence of activity traces in the Activity Trace Management component. These traces could be classified to labeled activity traces (LAT) which are already modeled in the seed ontologies or unlabeled activity traces (UAT) that do not exist in the seed activity ontologies.

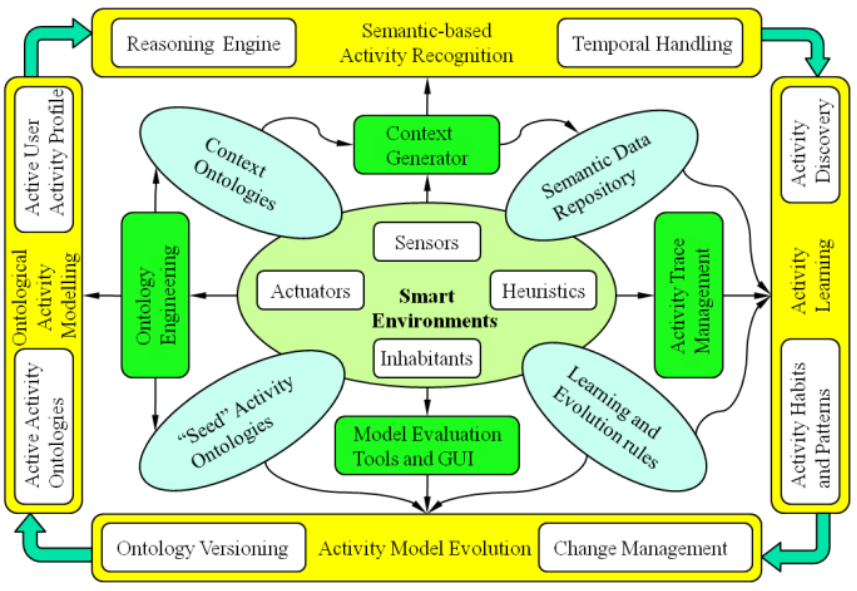

Figure 1. The integrated framework for activity modelling and recognition.

The purpose of the Activity Learning task is two-fold. Firstly it intends to identify the activities that a user performs which have not been modeled in the activity ontologies. Secondly, it is intended to learn the specific manner in which a user performs an activity. In this way, it can learn and adapt activity models in an evolutionary way according to a user's behavior, thus addressing the problems of knowledge-driven approach, i.e. model incompleteness and rigidity. The task is accomplished by mining the activity traces and time series of sensor data using learning rules in the Learning and Evolution Rules component.

The Activity Model Evolution task identifies changes that need to be made for the previous version of activity models based on the discovered and learnt activities. In addition, it recommends the locations and labels for these new activities in the hierarchy of the ADL ontologies. Model evolution is supported by ontology change management and versioning techniques. Human intervention is required to review and validate the changes for activity models, which is facilitated by the Model Evaluation Tools component. Once the current version of activity models is updated, it serves as the latest active activity models for future activity recognition.

The four key tasks interact with each other and form an integral lifecycle that can iterate indefinitely. As such, the framework enables and supports the following compelling features: a topdown approach to activity modelling and recognition, incremental activity discovery and learning, adaptive activity model evolution, activity recognition at both coarse-grained and fine-grained levels and increased accuracy of activity recognition. We have developed a semantic subsumption reasoning based activity recognition algorithm and semantic similarity based learning methods for both new activities and user profiles, and relevant supportive component. The details are described in [15] [16] [17].

\section{A SERVICE-ORIENTED ARCHITECTURE FOR ACTIVITY CONTEXT AWARENESS}

We proposed a service-oriented architecture as shown in Figure 2 to realize the presented systematic framework for activity monitoring, modelling and recognition. The underlying rationale is that rather than deploying a standalone activity context system in each smart home, activity recognition and application-specific functions based on activity context can be developed and deployed as services in cloud.

As can be seen in Figure 2, activity recognition and model learning can be implemented as services, and deployed in the cloud. These services can be accessed and (re)used by geographically distributed smart homes no matter where they are. Each smart home can collect its inhabitant's behavioral data through the deployed sensors and send the real-time sensor data to Data Services. Activity recognition is performed in the Activity Recognition Services which access sensor data stream via data services and using the Reasoning Services to conduct activity inference. The results of Activity Recognition Services, i.e. the activity context, can be used to support various domain-dependent application-specific functions. Some generic features are described below, to name but a few.

The generated activity traces from Activity Recognition Services will be stored in the Data Services and used by the Model Learning Services to learn new activity models or user profiles, which are used to update activity models.

Activity recognition services can identify activity anomaly or the assistance required during the performance of activities. Based on this a Reminder Prompting Service can provide activity assistance for the concerned inhabitants, such as context-aware just-in-time action prompting. Such assistances can be delivered to smart homes either in the form of a voice reminder through the service front-end user interface or control instructions which directly operate actuators to take appropriate actions such as closing window curtains.

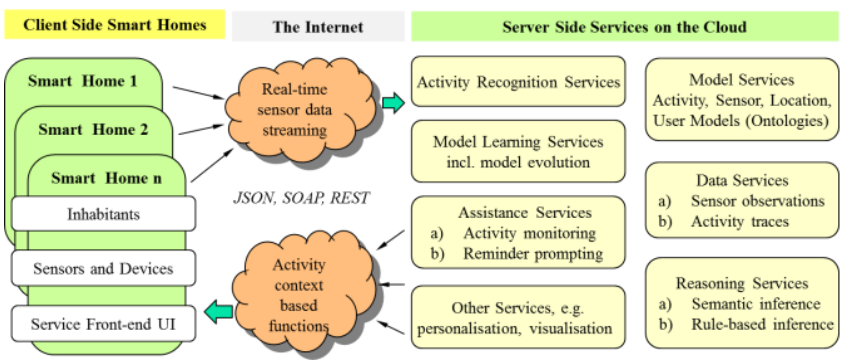

Figure 2. The service-oriented architecture for activity context awareness

The behavioral data collected by sensors within a smart home can be accumulated over time and analyzed using data mining and machine learning techniques to extract longitudinal activity patterns. Such patterns can be used by the Activity Monitoring Services to inform informal or formal care providers of the behavior changes or medical condition evolution of the monitored 
inhabitants. The presentation of patterns can be in the form of graphs and visualization, and delivered via a web based interface to the relevant stakeholders wherever they are located. For example, a person living in the UK can monitor their parent living in the Germany.

The SOA based architecture for the presented framework has several advantages. Firstly, the separation of the underlying hardware installation and configuration from high level data modelling and processing enabled by the SOA paradigm allow modular system design. This facilitates the rapid system development and deployment of assistive living solutions. Secondly, geographically distributed services in the cloud facilitate service reuse and sharing by many smart homes. In this paradigm system development amounts to the discovery and composition of services, thus allowing a large scale deployment and uptake of the ambient assisted living approach. Thirdly, a SOA is an open, extensible system architecture within which services can be added or removed as needed. As a result, a SOA based assistive living infrastructure can evolve incrementally with a increasing number of services to support more and rich functions and features. For example, personalization services can be added to address the uniqueness of each individual users in assistive living. Finally, a SOA implementation is platform neutral and technology independent. As such, it will be easy to assimilate and integrate existing technologies and prototypes developed and run on different platforms.

\section{IMPLEMENTATION and EVALUATION}

We have implemented the framework as both a standalone system and a SOA based system, which are described below.

\subsection{The Standalone System}

To test and evaluate the presented approach we have created the seed activity ontologies using the Protégé ontology editor [24] through knowledge engineering practice [15]. We have implemented a feature-rich standalone system for activity recognition and model learning as shown in Figure 3. The system was developed using C\#, ASP.NET, Ajax and Silverlight for audio and graphical user experience and deployed within our smart Lab [22]. The creation, management and query of semantic data was handled using the SemWeb semantic technologies for C\# [25] and custom queries. Semantic reasoning was implemented using the Euler [18] and Pellet [23] inference engines.

When an actor interacts with objects in sequence in real time, sensor activations are continuously fed into the system. Sensor data series are dynamically segmented [19] and recognition operations are repeatedly performed to carry out continuous, progressive activity recognition [10]. As depicted in Figure 3, the system can dynamically display the activated sensor sequence, the incrementally recognized activities and the system status in real time.

\subsection{The SOA-based System}

We also implemented the framework in a SOA architecture as shown in Figure 2. The current implementation of the system contains two supporting services, i.e. Data Services and Reasoning Services, and two core services, i.e. Activity Recognition Services and Assistance Services, each of them is described below.

The Reasoning Services provide inference capabilities for other services, in particular for activity recognition which is based semantic reasoning. The services integrate the semantic reasoning mechanisms offered by Pellet reasoning engine and a semantic repository provided by Jena. The semantic repository contains ontological activity models. In addition, other knowledge models such as personalization rules encoded by the semantic web rule language (SWRL) could also be included in terms of application scenarios.

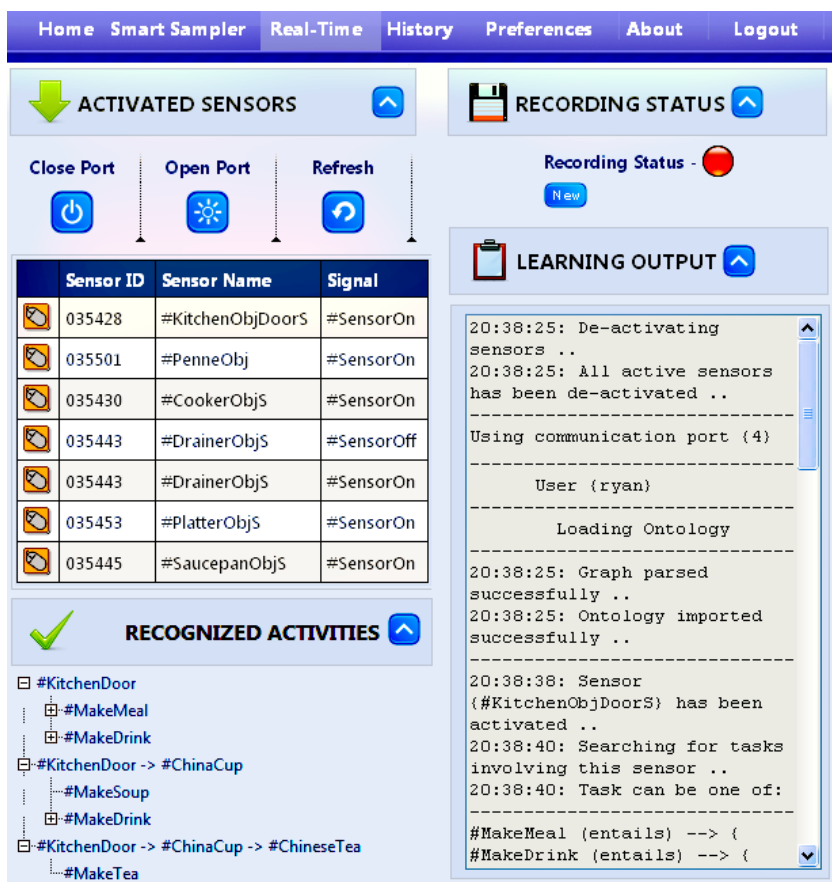

Figure 3. The system interface operating in real time mode. In the left-hand side, the top panel is used for communication port setup; the middle panel displays the sequence of activated objects; and the bottom panel presents progressively recognized activities in a tree-like hierarchy. In the right-hand side, the top panel contains function buttons for data recording and playback; the bottom panel presents a temporal trace of events during the system operation. The system can import activity ontologies, specify reasoning and learning parameters, select the modality of audio reminder, configure hardware and define event priorities and user activity profiles.

The Data Services provides facilities to record, maintain, handle raw sensor data and other intermediary data. These include sensor data, activity traces, status and state of operations and messages between web service requests. Application level functions can access various data through data services to conduct data mining and pattern recognition. The current version of Data Services is implemented using the $\mathrm{H} 2$ relational database management system and an SQL query based interface for data manipulation.

The Activity Recognition Services will take as inputs the streaming sensor data, ontological activity models and call reasoning services to infer ongoing activities. The inferred activity context can be used for assistance services to provide assistance or stored in Data Services for model learning and evolution through data mining. The activity recognition services are implemented as a Java based software which interacts with the backend reasoning services and the semantic repository within the reasoning services. 
The Assistance Services can provide various services based on application scenarios and application-specific features. For example, activity monitoring can show in real time a user's actions and activities and further based on the recognized behavior activity prompting service can provide just-in-time assistance to help user complete activities. Assistance services can be delivered through web based interfaces deployed directly in the users' smart homes or the care providers' office no matter where they are located. In current prototype we have developed a web based user interface for activity monitoring using only HTML, JavaScript, image files and CSS. No server side programming technologies are required to host an instance of this interface which reduces the complexity of this deployment. The interface uses AJAX technology to send and receive SOAP requests exclusively to and from various services.

The four services have been implemented using Java and deployed in Glassfish application server in two machines. One hosts the reasoning services and activity recognition services and the other the data services and activity monitoring services. Figure 4 shows the web based client side user interface of the implemented activity monitoring services and Figure 5 shows a screenshot of the real time monitoring of sensor activations.

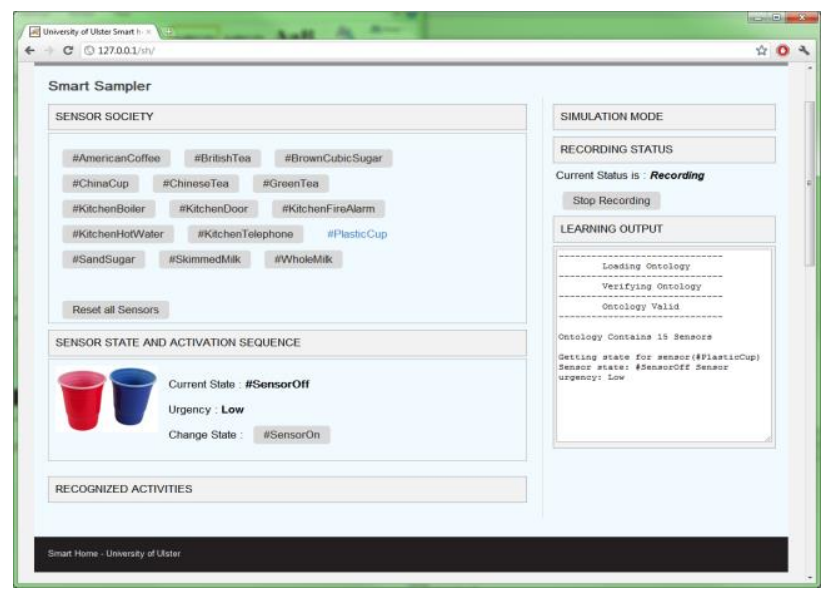

Figure 4. The Web based user interface for activity monitoring and recognition.

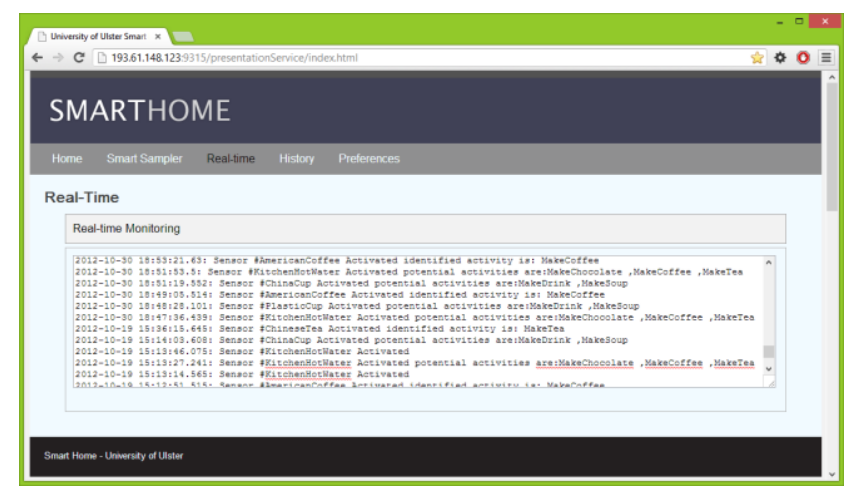

Figure 5. A screenshot of real time activity monitoring.

\section{TESTING AND ANALYSIS}

To systematically test and evaluate activity and profile learning, eight typical ADLs, including MakeCoffee, HaveBath, WatchTV,
MakeChocolate, BrushTeeth, MakeTea, BrushTeeth and MakePasta, were selected for the purposes of experimentation. For each activity, the required objects for performing the activity were identified and for each of them a contact sensor was attached. Each activity was designed to be performed in three different ways, leading to three different types of activity specification. The Type 1 activity specification, namely TP1 in short, can be viewed as the "standard" way of performing a specific activity. The Type 2 activity specification has the same set of objects; however, they are interacted with in a different order. The Type 3 activity specification has a different set of objects as it is intended to simulate noise on the sensor data, i.e. a faulty sensor by omitting a user-object interaction or a false sensor reading by adding an irrelevant object interaction.

Three actors took part in the experiments. Each of the participants interacts with the objects of each activity of the eight activities in accordance to the activity specifications for two rounds. This leads to a total of 3 (types) x 8 (activities) $\times 2$ (rounds) $\times 3$ (actors) $=144$ action traces.

\subsection{Results of the Standalone System}

We have used the standalone system to receive the sensor data stream from the aforementioned activity performance, and further conducted activity recognition. The recognition accuracies for type 1, 2 and 3 activity scenarios are $100 \%, 91.66 \%$ and $91.66 \%$ respectively with the overall recognition accuracy of $94.44 \%$. Detailed experiment analysis and discussion can be found in [16].

In order to test the activity learning capability we deliberately remove activity models, MakeChocolate and BrushTeeth, two of the eight selected activities from the seed activity ontologies. As a result the activity recognition of the system generated 100 LATs and 44 UATs. The analysis has shown that the semantic similarity based learning of new activities from UATs is $100 \%$ accurate. The result of learning object patterns that a user performs activities also reflects the ground truth accurately. Details can be found in [17].

\subsection{Results of the SOA based System}

The SOA based system was tested in the simulated kitchen environment of the SERG lab. The kitchen is equipped with wireless sensors which are connected to a local computer. The local computer hosts a networked sensor monitoring station and also the web based front-end user interface for activity monitoring as shown in Figure 6 and Figure 4 respectively. Services of the SOA architecture are all deployed in the same server which is located in a different computer in a different location, thus operating in a distributed manner suitable for deployment across the internet.

Sensor activations are collected by the sensor monitoring station which in turn sends records of these sensor observations to the activity recognition service via the enterprise service bus. The recognition service interacts with other service nodes to perform its reasoning process and reports the outcome to both the data service node and the sensor monitoring station. All sensor activations were logged along with the activities that were recognized.

During the testing a researcher was given the task of performing multiple simulated activities of daily living that would be likely take place in a kitchen setting. This simulation involved interacting with the necessary objects required to complete the task but without creating the end product. 


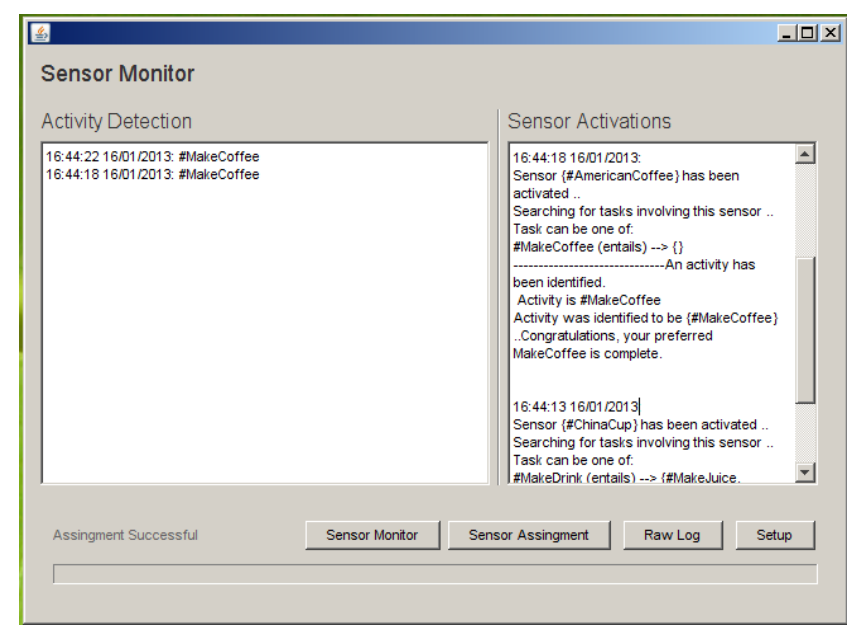

Figure 6. The sensor monitoring station in use during svstem testing and evalution

Given that the services of the SOA architecture are mainly the wrapping of the existing functions / technologies which have already tested and evaluated in the standalone system, the emphasis of the testing for the SOA system is not the performance of the underlying technologies but the feasibility and effectiveness of the SOA approach. As a result the testing focused predominately on the communication and interactions between services, i.e. the sending and receipts of sensor data activations and various messages exchanged between services.

Table 1. Results of testing the SOA-based implementation

\begin{tabular}{|l|l|l|l|}
\hline Functionality Tested & Permutations & $\begin{array}{l}\text { Successful } \\
\text { comparisons }\end{array}$ & $\begin{array}{l}\text { Succes } \\
\text { s rate }\end{array}$ \\
\hline Sensor activations & 220 & 220 & $100 \%$ \\
\hline $\begin{array}{l}\text { Reasoning results from } \\
\text { sensor operations }\end{array}$ & 220 & 220 & $100 \%$ \\
\hline $\begin{array}{l}\text { Addition of activity } \\
\text { preferences }\end{array}$ & 40 & 40 & $100 \%$ \\
\hline $\begin{array}{l}\text { Removal of activity } \\
\text { preferences }\end{array}$ & 40 & 40 & $100 \%$ \\
\hline Retrieval of logs & 100 & 100 & $100 \%$ \\
\hline Deletion of logs & 100 & 100 & $100 \%$ \\
\hline Creation of logs & 100 & 100 & $100 \%$ \\
\hline $\begin{array}{l}\text { Appending information to } \\
\text { logs }\end{array}$ & 400 & 400 & $100 \%$ \\
\hline $\begin{array}{l}\text { Storage, retrieval and } \\
\text { comparison of data key } \\
\text { pairs from the data service }\end{array}$ & 1024 & 1024 & $100 \%$ \\
\hline
\end{tabular}

The testing used three activities MakeCoffee, MakeBritishTea, and MakeGreenTea. Each of them is performed multiple times over the course of a day at different rates and in varying manners. Sensor activations and messages are recorded and analyzed. The results in Table I show that all operations are performed and activities are recognized correctly. The system behaves reliably and identically to the standalone system.

\section{CONCLUSIONS}

This paper introduces a generic framework for activity modeling and recognition and its associated implementation, in particular, a SOA based system. The focus of the paper is not on the underlying modelling, algorithms and methods but on 1) the iterative process and mechanisms that support incremental model learning and evolution, thus improving activity recognition; and 2) a SOA based system implementation that facilitates rapid system development and large scale deployment, thus supporting reusability and scalability. The paper described the key components and their interplay, with particular emphasis being placed on the SOA based technology infrastructure. Experiments, testing and evaluation have been conducted and initial results have shown the framework and SOA based infrastructure are working. We believe the presented framework and SOA provide a realistic industry-strength solution to activity context aware applications. The future work will be the full implementation and large scale evaluation of the SOA system.

\section{REFERENCES}

[1] Weiser, M. 2002. The computer for the 21st century (reprint). Pervasive Computing, vol.1, no.1, pp.19-25.

[2] Bettini, C., Brdiczka, O., Henricksen, K., Indulska, J., Nicklas, D., Ranganathan, A. and Riboni, D. 2010. A survey of context modelling and reasoning techniques. Pervasive and Mobile Computing, vol. 6, pp.161-180.

[3] Hoey, J. 2007. Little Value-Directed Human Behavior Analysis from Video Using Partially Observable Markov Decision Processes. IEEE Transactions on Pattern Analysis and Machine Intelligence, vol.29, no.7, pp.1118-1132.

[4] Moeslund, T.B., Hilton, A., Krüger, V. 2006. A survey of advances in vision-based human motion capture and analysis. Comput. Vis. Image Understand., vol.104, no.2, pp.90-126.

[5] Fiore, L., Fehr, D., Bodor, R., Drenner, A., Somasundaram, G., Papanikolopoulos, N. 2008. Multi-Camera Human Activity Monitoring. Journal of Intelligent and Robotic Systems, vol.52, no.1, pp.5-43.

[6] Turaga, P., Chellappa, R., Subrahmanian, V.S., Udrea, O. 2008. Machine Recognition of Human Activities: A Survey. IEEE Transaction on Circuits and Systems for Vedio Technology, Vol.18, No.11, pp.1473-1488.

[7] Lee, S.W., Mase, K. 2002. Activity and location recognition using wearable sensors. IEEE Pervasive Computing, vol.1, no.3, pp.24-32.

[8] Parkka, J., Ermes, M., Korpipaa, P., Mantyjarvi, J., Peltola, J., Korhonen, I. 2006. Activity classification using realistic data from wearable sensors. IEEE Transactions on Information Technology in Biomedicine, vol.10, no.1, pp.119-128.

[9] Philipose, M., Fishkin, K.P., Perkowitz, M., Patterson, D.J., Fox, D., Kautz, H., Hahnel, D. 2004. Inferring activities from interactions with objects. IEEE Pervasive Computing, pp.5057.

[10] Baader, F., Calvanese, D., McGuinness, D.L. 2003. The Description Logic Handbook: Theory, Implementation, Applications, Cambridge University Press, ISBN 0-52178176-0. 
[11] Bao, L. and Intille, S. 2004. Activity recognition from user annotated acceleration data, In Proc. Pervasive, LNCS3001, pp.1-17.

[12] Bouchard, B. and Giroux, S. 2006. A Smart Home Agent for Plan Recognition of Cognitively-impaired Patients, Journal of Computers, vol.1, no.5, pp.53-62, 2006.

[13] Brdiczka, O. and Crowley, J.L. 2009. Learning situation models in a smart home", IEEE Transactions on Systems, Man and Cybernetics - Part B: Cybernetics, vol.39, no.1, pp.56-63.

[14] Chen, L., Nugent, C., Mulvenna, M. and Finlay, D. 2008. A Logical Framework for Behaviour Reasoning and Assistance in a Smart Home, International Journal of Assistive Robotics and Mechatronics, vol.9, no.4, pp.20-34.

[15] Chen, L. and Nugent, C.D. 2009. Semantic Data Management for Situation-aware Assistance in Ambient Assisted Living, In the proceedings of the 11th International Conference on Information Integration and Web-based Applications and Services (iiWAS2009), pp.296-303.

[16] Chen, L., Nugent, C.D. and Wang, H. 2012. A KnowledgeDriven Approach to Activity Recognition in Smart Homes, IEEE Transactions on Knowledge and Data Engineering, vol.24, no.6, pp961-974.

[17] Chen, L., Nugent, C.D. and Okeyo, G. 2013. An Ontologybased Hybrid Approach to Activity Modeling for Smart Homes, IEEE Transactions on Human-Machine Systems (THMS), to appear.

[18] Euler proof mechanism, www.agfa.com/w3c/euler/
[19] Okeyo, G., Chen, L., Wang, H. and Sterritt, R. 2013. Dynamic Sensor Data Segmentation for Real time Activity Recognition, Pervasive and Mobile Computing, DOI=http://dx.doi.org/10.1016/j.pmcj.2012.11.004.

[20] Helal, S., Mann, W., El-Zabadani, H., King, J., Kaddoura, Y. and Jansen, E. 2005. The Gator Tech Smart House: a programmable pervasive space, Computer, vol.38, no.3, pp. $50-60$.

[21] Hoey, J. and Poupart, P. 2005. Solving POMDPs with continuous or large discrete observation spaces, In Proc. Int'l. Joint Conf. on Artificial Intelligence, pp.1332-1338.

[22] Nugent, C.D. and Mulvenna, M. 2009. Experiences in the Development of a Smart Lab, The International Journal of Biomedical Engineering and Technology, vol.2, no.4, pp.319-331.

[23] Pellet: OWL 2 Reasoner for Java, http://clarkparsia.com/pellet

[24] The Protégé framework, http://protege.stanford.edu

[25] Semantic Web RDF Library for C\#.NET, http://razor.occams.info/code/semweb/

[26] Sanchez, D. and Tentori, M. 2008. Activity recognition for the smart hospital, IEEE Intelligent Systems, vol.23, no.2, pp.50-57.

[27] Ye, J., Stevenson, G. and Dobson, S. 2011. A top-level ontology for smart environments, Pervasive and Mobile Computing, vol.7, no.3, pp.359-378. 